\title{
Perfil clínico de 62 casos de distúrbios da diferenciação sexual
}

\author{
Clinical profile of 62 cases of sexual differentiation disorders
}

\begin{abstract}
Juliana Gabriel R. de Andrade ${ }^{1}$, Rosa Rita S. Martins ${ }^{2}$, Dayse Caldas ${ }^{3}$, Jucimar Brasil ${ }^{4}$, André Luiz A. Meiriño ${ }^{5}$, Monica de Paula Jung $^{6}$
\end{abstract}
\section{RESUMO}

Objetivo: Descrever o perfil clínico dos casos de distúrbios da diferenciação sexual em acompanhamento no Instituto Estadual de Diabetes e Endocrinologia Luiz Capriglione, no Rio de Janeiro, nos últimos cinco anos.

Métodos: Revisão dos prontuários dos pacientes, com o diagnóstico de genitália ambígua em acompanhamento nos últimos cinco anos, segundo os critérios clínicos descritos por Danish, em 1982. O registro mais antigo foi feito em 1981 e o mais recente de junho de 2006.

Resultados: Foram encontrados 62 casos de genitália ambígua: 26 com registro do sexo feminino e 36 com registro do sexo masculino. O diagnóstico mais freqüente foi o de hiperplasia congênita de supra-renal $(33,9 \%)$, seguido de quadros sindrômicos $(14,5 \%)$ e disgenesias gonadais (9,7\%). A média de idade ao diagnóstico foi de 7,2 anos (de zero a 42 anos).

Conclusões: A ambigüidade genital não é uma doença específica, mas um conjunto de alterações que direcionam o clínico a buscar diagnósticos específicos. A frequiência dessa afecção depende dos critérios diagnósticos utilizados. A adoção de critérios amplos aumenta a chance de detecção precoce do quadro bem como de cuidado adequado a crianças com distúrbios da diferenciação sexual.

Palavras-chave: genitália; diferenciação sexual; etiologia.

\section{ABSTRACT}

Objective: To report patients with ambiguous genitalia assisted at the State Institute of Diabetes and Endocrinology of Rio de Janeiro, Brazil, in the last five years.

Methods: Retrospective chart review of all cases of ambiguous genitalia, classified according to Danish criteria (1982), who attended follow-up visits in the last five years. The oldest record is from 1981 and the most recent one, 2006.

Results: 62 patients with ambiguous genitalia were found: 26 of them assigned as females and 36 as males. The most frequent diagnosis was congenital adrenal hyperplasia (33.9\%), followed by syndromic diseases (14.5\%) and gonadal dysgenesis (9.7\%). The majority of patients with ambiguous genitalia were detected at birth, however, the mean age at the diagnosis was 7.2 years (zero to 42 years).

Conclusions: Genital ambiguity is not a specific disease, but a set of problems that directs the physician to search specific diagnosis. The frequency of this condition depends on the diagnostic criteria used. Adopting amplified criteria in order to diagnose genital ambiguity will increase the possibility of early detention and adequate handling of these patients.

Key-words: genitalia; sexual differentiation; etiology.
Instituição: Instituto Estadual de Diabetes e Endocrinologia Luiz Capriglione, Rio de Janeiro, RJ, Brasil

'Endocrinologista, aluna de pós-graduação em nível de mestrado da Universidade Estadual de Campinas, Campinas, SP, Brasil

${ }^{2}$ Geneticista, doutora em Ciências Biológicas pela Universidade Federal do Rio de Janeiro (UFRJ), Rio de Janeiro, RJ, Brasil

${ }^{3}$ Endocrinologista, doutora em Endocrinologia e Metabologia pela UFRJ, Rio de Janeiro, RJ, Brasil

${ }^{4}$ Endocrinologista chefe do Ambulatório de Andrologia do Instituto Estadual de Diabetes e Endocrinologia Luiz Capriglione, Rio de Janeiro, RJ, Brasil ${ }^{5}$ Endocrinologista, Rio de Janeiro, RJ, Brasil

${ }^{6}$ Endocrinologista, mestre em Ciências pelo Instituto Fernandes Figueira da Fundação Oswaldo Cruz, Rio de Janeiro, RJ, Brasil
Endereço para correspondência:

Juliana Gabriel R. de Andrade

Rua Amaury Filho, 77

CEP 22790-320 - Rio de Janeiro/RJ

E-mail: jugabrielra@yahoo.com.br

Recebido em: 11/4/2008

Aprovado em: 6/7/2008 


\section{Introdução}

Deparar-se com um caso de ambigüidade genital é uma situação difícil para toda a equipe de saúde que dá assistência à criança recém-nascida. Vários aspectos devem ser levados em consideração na abordagem, desde o diagnóstico diferencial, passando pela designação sexual que, se equivocada, pode ter repercussões importantes tanto psicológicas quanto sociais para a família e por toda a vida da criança ${ }^{(1)}$; há ainda a complexidade da fisiopatologia, a grande diversidade de apresentações clínicas e o vasto leque de possibilidades diagnósticas, além das implicações prognósticas.

A caracterização precoce de um paciente com alterações na diferenciação sexual possibilita a descoberta de situações cruciais para a vida e para a sobrevida do paciente, como, por exemplo, uma crise adrenal, a presença de gonadoblastoma em algumas disgenesias gonadais e de outros tumores, como o de Wilms. Como a apresentação clínica é bastante variável, podendo ser sutil a ponto de passar despercebida inicialmente, é necessário o uso de critérios para o diagnóstico que, quanto mais amplos, maior a chance de detecção dessas situações.

Ainda existem muitas controvérsias na literatura acerca da nomenclatura mais adequada e da definição dos melhores critérios diagnósticos. Por esse motivo, o tema "ambigüidade genital" vem sendo bastante estudado pela comunidade científica e, a cada ano, são realizados novos estudos que tratam não somente da fisiopatologia mas também da investigação diagnóstica, conduta, aspectos psicológicos e epidemiológicos. Ainda assim existem poucos dados acerca de casuísticas brasileiras.

Procurou-se, neste trabalho, avaliar o perfil clínico dos pacientes caracterizados como portadores de ambigüidade genital em acompanhamento nos últimos cinco anos no Instituto Estadual de Diabetes e Endocrinologia Luiz Capriglione (Iede), Rio de Janeiro (RJ), Brasil.

\section{Métodos}

Foram selecionados todos os prontuários dos pacientes encaminhados ao hospital com suspeita ou diagnóstico de ambigüidade genital, criptorquidia e micropênis, que estivessem em acompanhamento nos últimos cinco anos. Para tanto, foram levantados dados do arquivo nosológico do Ambulatório de Genética e do Laboratório de Citogenética, bem como dos arquivos dos Ambulatórios de Hipófise/Supra-Renal, Andrologia e Endocrinologia Pediátrica.
O estudo foi aprovado pelo Comitê de Ética em Pesquisa com seres humanos do Iede e a coleta de dados foi realizada de março a julho de 2006. Foram localizados 67 prontuários e cinco deles foram excluídos por não preencherem os critérios propostos para a caracterização da genitália ambígua. O registro mais antigo foi do ano de 1981 e o mais recente de junho de 2006.

O critério de inclusão utilizado foi o preenchimento dos requisitos clínicos de Danish ${ }^{(2)}$ para caracterizar a ambigüidade genital. Nessa classificação, há diagnóstico de ambigüidade genital se qualquer manifestação, das listadas a seguir, estiverem presentes no paciente ao nascimento:

- em genitália de aspecto masculino: gônadas não palpáveis; tamanho peniano esticado menor que 2,5 desvios-padrão da média de tamanho peniano normal para a idade; gônadas pequenas, ou seja, maior diâmetro inferior a $8 \mathrm{~mm}$; presença de massa inguinal palpável (que poderá corresponder a útero e trompas rudimentares) e hipospádia.

- em genitália de aspecto feminino: diâmetro clitoriano superior a $6 \mathrm{~mm}$; gônada palpável em bolsa labioescrotal; fusão labial posterior; massa inguinal (que possa corresponder a testículos).

Excluíram-se os pacientes cuja caracterização da genitália não se enquadrava nesses requisitos, ainda que o paciente fosse portador de alguma entidade mórbida que pudesse cursar com ambigüidade.

Foram coletados os seguintes dados dos prontuários: demografia (naturalidade, residência atual, sexo de registro, data de nascimento); características clínicas (exame clínico da genitália e classificação de Prader, presença de outras anomalias ou dismorfias, exames laboratoriais ou de imagem); dados de história clínica (idade em que se iniciaram os sintomas, história neonatal, relato de crise adrenal ou de hipertensão, história familiar, gestacional e relato de fertilidade). Foi considerada história familiar positiva a presença de: relato familiar de genitália ambígua; crise adrenal e/ou morte neonatal; infertilidade; criptorquidia, hipospádia e/ ou micropênis e síndromes semelhantes à apresentada pelo paciente. Além disso, coletaram-se informações sobre o cariótipo do paciente e sobre cirurgia de reparação genital. Vale ressaltar que a coleta de dados limitou-se à consulta de informações contidas nos prontuários.

Para análise descritiva dos dados obtidos, utilizou-se o software Epi Info ${ }^{\mathrm{TM}}$ Versão 3.3.2, obtida gratuitamente através do portal do National Center of Health Statistics (NHCS) dos Estados Unidos da América. 


\section{Resultados}

Dos 67 prontuários obtidos, cinco foram excluídos por não preencherem os requisitos para o diagnóstico de genitália ambígua: um caso de disgenesia gonadal pura 46 $\mathrm{XY}$ com fenótipo completamente feminino; um paciente suspeito de micropênis que era, na realidade, limite inferior da normalidade; um caso de suspeita de clitoromegalia normal para um recém-nascido pré-termo; um paciente com microrquia e ginecomastia e um caso sem diagnóstico. Dos 62 prontuários restantes, os diagnósticos estão descritos na Tabela 1.

Dentre os pacientes estudados, $52(83,9 \%)$ eram do Rio de Janeiro, quatro $(6,5 \%)$ de outros estados. Em cinco $(8,1 \%)$ não havia relato da naturalidade no prontuário. Dos 62 pacientes, apenas sete não tinham cariótipo. Dentre os 53 restantes, só três apresentavam anomalias cromossômicas (Tabela 2).

$\mathrm{Na}$ maioria, houve coincidência entre o sexo genético e o sexo social, havendo apenas três $(4,8 \%)$ casos de cariótipo $\mathrm{XX}$ e registro masculino e dois $(3,2 \%)$ casos de cariótipo $\mathrm{XY}$ entre os pacientes de registro feminino.

A distribuição quanto ao estadiamento de Prader, de acordo com o sexo de registro, demonstrou que, dentre os pacientes identificados como femininos, a maioria obteve Prader 3 e nenhum obteve Prader 5, enquanto nos registrados como masculinos, a maioria obteve Prader 5 e nenhum foi classificado como Prader 1 ou 2 (Gráfico 1).

Tabela 1 - Freqüência do diagnóstico nos 62 casos estudados

\begin{tabular}{lcc}
\hline Diagnóstico & Número de casos & Freqüência (\%) \\
\hline Anorquia & 4 & 6,4 \\
Criptorquidia isolada & 4 & 6,4 \\
Criptorquidia associada a micropênis ou hipospádia & 2 & 3,2 \\
Hipospádia isolada & 3 & 4,9 \\
Hipospádia associada à micropênis & 1 & 1,6 \\
Micropênis isolado & 3 & 4,9 \\
Disgenesias gonadais & 6,7 \\
Hermafroditismo verdadeiro & 6 & 1,6 \\
Homem XX & 1 & 3,2 \\
Hiperplasia adrenal congênita* & 2 & 33,9 \\
Doenças hipofisárias & 21 & 4,9 \\
Síndromes específicas* & 3 & 14,5 \\
Síndrome de resistência androgênica & 3 & 3,2 \\
Clitoromegalia isolada & 9 & 1,6 \\
Total & 2 & $\mathbf{1 0 0}$ \\
\hline
\end{tabular}

*20 casos deficiência de 21 hidroxilase e um de deficiência de 11 beta hidroxilase; **inclui síndrome de Noonan, síndrome de Kallman, síndrome de Peters-Plus, síndrome de Prune-Belly, síndrome de Smith-Lemli-Opitz e síndrome da deleção do 18q.
A história familiar de genitália ambígua, crise adrenal ou morte neonatal foi positiva em $46,2 \%$ dos pacientes com adrenal congênita. Nos pacientes com de registro masculino, apenas $14 \%$ tinham história familiar positiva, sendo um homem XX, um caso de Síndrome de Smith-Lemli-Opitz com hipogonadismo hipogonadotrófico isolado, pan-hipo-

Tabela 2 - Pacientes com genitália ambígua e anomalias cromossômicas

\begin{tabular}{lcl}
\hline Paciente & Cariótipo & Diagnóstico \\
\hline 26 & $45, \mathrm{X} / 46, \mathrm{X}$ dicYq & Disgenesia gonadal mista \\
42 & $46, \mathrm{XY}$ del 18q22 & Deleção do 18q \\
49 & $46, \mathrm{XY}$ inv 18 & Deleção do 18q (suspeita) \\
\hline
\end{tabular}

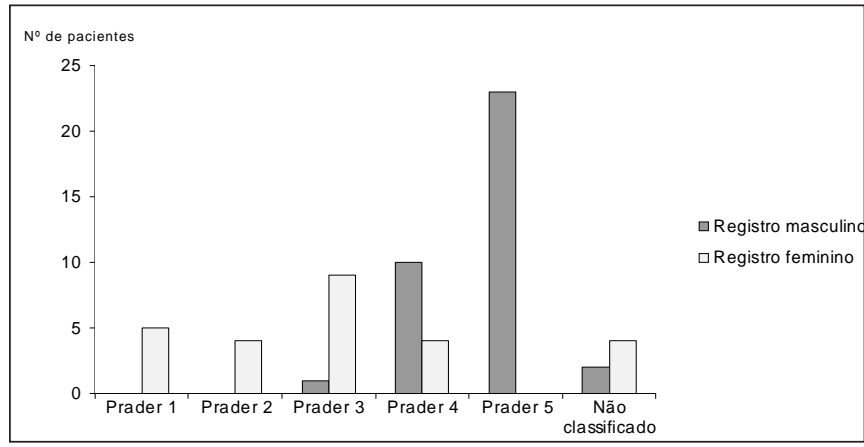

Gráfico 1 - Estadiamento de Prader, de acordo com o sexo de registro, os 62 casos analisados. registro do sexo feminino, sendo todos os casos de hiperplasia 
pituitarismo e criptorquidia bilateral. No total, o histórico familiar positivo compreendeu $27,4 \%$ dos casos.

A média de idade ao diagnóstico foi de 7,2 anos, com mediana de três anos e variação de zero a 42 anos. A maioria dos casos de atraso ao diagnosticar a ambigüidade foi devido à dificuldade de acesso ao sistema de saúde (por exemplo, pelo fato de o paciente vir do interior). No caso de alterações como micropênis e criptorquidia, a maioria foi encaminhada para avaliação próxima à puberdade. A lista com todos os casos estudados está expressa na Tabela 3.

\section{Discussão}

O diagnóstico de um caso de "intersexo" traz consigo uma série de questionamentos por parte da família do paciente. Vários aspectos devem ser levados em consideração no atendimento a esses indivíduos, e isso não inclui apenas a determinação do sexo, mas também as implicações sociais, psicológicas e legais. Por esse motivo, tais pacientes devem ser acompanhados em serviço com equipe multidisciplinar especializada formada por clínicos (pediatras ou endocrinologistas), cirurgiões, médicos-legistas e outros profissionais, como psicólogos e assistentes sociais ${ }^{(3)}$.

Os crescentes avanços na área da genética e biologia molecular vêm possibilitando a elucidação diagnóstica de um número cada vez maior de casos, o que também permite o aconselhamento genético para portadores assintomáticos de mutações, como a hiperplasia adrenal congênita ${ }^{(4)}$, a deficiência de 5-alfa redutase e a síndrome de insensibilidade aos androgênios. A biologia molecular permite também uma avaliação prognóstica desses pacientes quanto ao risco de tumor $^{(5)}$ e à possibilidade de fertilidade. O propósito deste estudo, contudo, não é abordar o manejo dessas afecções.

A definição "genitália ambígua" é ainda controversa na literatura. Alguns caracterizam a ambigüidade genital por sua concepção mais literal, sendo classificados como tal apenas os pacientes cujo sexo não se pôde designar na avaliação inicial. Entretanto, outros autores já começam a classificar os pacientes não como portadores de "ambigüidade" ou "intersexo" e, sim, como portadores de Distúrbios da Diferenciação Sexual (DDS), tendo ou não alterações genitais extremas a ponto da designação sexual estar comprometida ${ }^{(6,7)}$.

A ambigüidade genital ainda é considerada rara no meio médico. No entanto, estudos sobre prevalência mostram resultados conflitantes. Um dos pontos que poderia explicar tal diferença está no critério utilizado para caracterizar a ambigüidade genital.
Castilla et al, em $1987^{(8)}$, tentou avaliar a prevalência em nosso meio. O estudo buscou revisar o perfil epidemiológico da genitália ambígua na América do Sul, incluindo 70 hospitais de referência em 34 cidades de nove países sul-americanos, entre 1967 e 1982. Considerou-se a ambigüidade genital quando não houve possibilidade de definir o sexo do recém-nascido. Foram incluídas crianças com genitália ambígua, malformada ou ausente, mas outros graus de alteração fenotípica não foram levados em consideração. A prevalência isolada de genitália ambígua foi por volta de 1 a cada 20 mil nascimentos, sendo a hiperplasia adrenal congênita sua principal causa (25 a 50\% da amostra). Quando associada a outras malformações, a prevalência de genitália ambígua aumentou para 1:6.900 do total de nascimentos. Como críticas a este estudo, argumenta-se que a definição de genitália ambígua não incluiu hipospádia e o tempo de observação dos pacientes foi de apenas três dias, ou seja, somente foram analisados os recém-nascidos cuja genitália não pôde ser identificada como feminina ou masculina na primeira avaliação.

Outro artigo sobre o perfil etiológico foi o publicado por Al-Mutair et $a^{(9)}$. Este estudo avaliou 120 casos com diagnóstico de ambigüidade genital encaminhados ao laboratório de citogenética, entre 1989 e 1999. O diagnóstico era feito com base no cariótipo e na impressão clínica inicial do médico assistente, pediatra ou endocrinologista. Dos 120 pacientes selecionados, $63(52,5 \%)$ apresentavam causas endócrinas, sendo $41(34,1 \%)$ de hiperplasia adrenal congênita. Os 57 pacientes restantes foram classificados como portadores de defeitos congênitos e incluíam anormalidades de cloaca, defeitos metabólicos, síndromes específicas e hipospádia.

Em 2000, Blackless et al ${ }^{(7)}$ publicaram uma revisão da literatura de 1955 a 2000 sobre a frequiência de desvio do 'ideal' de homem e mulher. Foi feita uma descrição detalhada do que seria considerado o 'homem perfeito' e a 'mulher perfeita'; dessa forma, tudo aquilo que se desviasse de tal definição seria caracterizado como DDS. Praticamente todos os casos de alteração da diferenciação sexual foram incluídos, inclusive distúrbios cromossômicos, agenesia de pênis e vagina, criptorquidia, hipospádia, micropênis e clitoromegalia isolados, distúrbios na produção hormonal, causas tumorais de excesso hormonal, disgenesias gonadais e hermafroditismo verdadeiro. Estima-se que a freqüência de distúrbios da diferenciação sexual poderia ser tão alta quanto $2 \%$ dos nascidos vivos. A frequiência de 
Tabela 3 - Sexo de registro, cariótipo, apresentação clínica, diagnóstico e idade ao diagnóstico

\begin{tabular}{|c|c|c|c|c|c|c|}
\hline Caso & Registro & Cariótipo & GA & Apresentação Clínica & Diagnóstico & $\begin{array}{l}\text { Idade } \\
\text { (anos) }\end{array}$ \\
\hline 1 & $\mathrm{~F}$ & $46, X Y$ & $\mathrm{~S}$ & Massa supra-púbica & $\begin{array}{l}\text { Síndrome de Resistência } \\
\text { Androgênica Completa }\end{array}$ & 42 \\
\hline 2 & M & $46, X Y$ & $\mathrm{~S}$ & Criptorquidia bilateral & Anorquia bilateral & 5 \\
\hline 3 & $\mathrm{~F}$ & $46, X Y$ & $\mathrm{~S}$ & Massa inguinal & Disgenesia gonadal $46, X Y$ & $?$ \\
\hline 4 & M & $46, X Y$ & $\mathrm{~S}$ & Criptorquidia bilateral & Disgenesia testicular & 11 \\
\hline 5 & $\mathrm{~F}$ & CSP & $\mathrm{S}$ & $\begin{array}{l}\text { Clitoromegalia + } \\
\text { orifício urogenital único }\end{array}$ & Deficiência 21 hidroxilase & 0,1 \\
\hline 6 & $\mathrm{~F}$ & - & $\mathrm{S}$ & $\begin{array}{l}\text { Clitoromegalia + } \\
\text { orifício urogenital único }\end{array}$ & Deficiência 21 hidroxilase & 6 \\
\hline 7 & $\mathrm{~F}$ & - & $\mathrm{S}$ & Clitoromegalia & Deficiência 21 hidroxilase & 0,1 \\
\hline 8 & M & - & $\mathrm{S}$ & Micropênis & Micropênis & 14 \\
\hline 9 & M & $46, X Y$ & $\mathrm{~S}$ & $\begin{array}{l}\text { Micropênis + } \\
\text { criptorquidia bilateral }\end{array}$ & Pan-hipopituitarismo & 11 \\
\hline 10 & $\mathrm{~F}$ & $46, X X$ & $\mathrm{~S}$ & Clitoromegalia isolada & Hiperplasia de clitóris & 6 \\
\hline 11 & $\mathrm{~F}$ & - & $\mathrm{S}$ & $\begin{array}{l}\text { Clitoromegalia }+ \\
\text { orifício urogenital único }\end{array}$ & Deficiência 21 hidroxilase & 0,1 \\
\hline 12 & M & $46, X Y$ & $\mathrm{~S}$ & Hipospádia & Smith-Lemli-Opitz (suspeita) & 0,1 \\
\hline 13 & M & $46, X Y$ & $\mathrm{~S}$ & Criptorquidia bilateral & Anorquia bilateral & 3 \\
\hline 14 & M & - & $\mathrm{S}$ & Micropênis & Micropênis & 11 \\
\hline 15 & M & $46, X Y$ & $\mathrm{~S}$ & Criptorquidia bilateral & Síndrome de Noonan (suspeita) & 0,1 \\
\hline 16 & M & $46, X Y$ & $\mathrm{~S}$ & Criptorquidia bilateral & Anorquia Bilateral & 14 \\
\hline 17 & $\mathrm{~F}$ & - & $\mathrm{S}$ & $\begin{array}{l}2 \text { hemibolsas com gônadas } \\
\text { palpáveis + orifício urogenital } \\
\text { único + falus de } 2 \mathrm{~cm}\end{array}$ & $\begin{array}{l}\text { Síndrome de Resistência } \\
\text { Androgênia Parcial (suspeita) }\end{array}$ & 1 \\
\hline 18 & M & $46, X Y$ & $\mathrm{~S}$ & Criptorquidia bilateral & Criptorquidia bilateral & 0,1 \\
\hline 19 & M & $46, X Y$ & S & Hipospádia & Hipospádia (prematuridade?) & 0,1 \\
\hline 20 & $\mathrm{~F}$ & - & $\mathrm{S}$ & Clitoromegalia & Deficiência 21 hidroxilase & 0,1 \\
\hline 21 & $\mathrm{~F}$ & - & $\mathrm{S}$ & Clitoromegalia & Deficiência 21 hidroxilase & 13 \\
\hline 22 & M & - & $\mathrm{S}$ & Criptorquidia bilateral & Criptorquidia bilateral + micropênis & 9 \\
\hline 23 & M & $46, X Y$ & $\mathrm{~S}$ & $\begin{array}{l}\text { Micropênis + } \\
\text { Criptorquidia bilateral }\end{array}$ & Criptorquidia bilateral & 9 \\
\hline 24 & M & CSN & $\mathrm{S}$ & Criptorquidia bilateral & Disgenesia testicular & 27 \\
\hline 25 & $\mathrm{~F}$ & - & $\mathrm{S}$ & Clitoromegalia & Deficiência 21 hidroxilase & 8 \\
\hline 26 & $\mathrm{~F}$ & $45, \mathrm{X0} / 46, \mathrm{X}$ dic Yq & $\mathrm{S}$ & $\begin{array}{l}\text { Seio urogenital único + } \\
1 \text { gônada palpável em } \\
\text { hemibolsa direita + falus } \\
\text { hipertrofiado }\end{array}$ & Disgenesia gonadal mista & 0,1 \\
\hline 27 & $\mathrm{~F}$ & $46, X X$ & $\mathrm{~S}$ & Clitoromegalia & Deficiência 21 hidroxilase & 0,1 \\
\hline 28 & M & $46, X Y$ & $\mathrm{~S}$ & $\begin{array}{l}\text { Hipospádia }+ \\
\text { Criptorquidia bilateral }\end{array}$ & Síndrome de Peters-Plus & 1 \\
\hline 29 & $\mathrm{~F}$ & - & $\mathrm{S}$ & Pós-cirurgia corretiva & Deficiência 21 hidroxilase & 5 \\
\hline 30 & $\mathrm{~F}$ & - & $\mathrm{S}$ & $\begin{array}{l}\text { Clitoromegalia }+ \\
\text { pseudo seio urogenital }\end{array}$ & Deficiência 21 hidroxilase & 0,1 \\
\hline 31 & M & - & $\mathrm{S}$ & Micropênis & Micropênis & 10 \\
\hline 32 & $\mathrm{M}$ & $46, X Y$ & $\mathrm{~S}$ & Micropênis + hipospádia & Hipospádia & 0,5 \\
\hline
\end{tabular}

GA: genitália ambígua; N: não; S: sim; CSP: cromatina sexual positiva; CSN cromatina sexual negativa. 
Continuação (Tabela 3)

\begin{tabular}{|c|c|c|c|c|c|c|}
\hline Caso & Registro & Cariótipo & GA & Apresentação Clínica & Diagnóstico & Idade \\
\hline 33 & $\mathrm{M}$ & $46, X Y$ & $\mathrm{~S}$ & $\begin{array}{l}\text { Micropênis + } \\
\text { criptorquidia bilateral }\end{array}$ & Disgenesia testicular & 1 \\
\hline 34 & M & $46, X Y$ & $S$ & $\begin{array}{l}\text { Micropênis + } \\
\text { criptorquidia bilateral }\end{array}$ & Pan - hipopituitarismo & 0,4 \\
\hline 35 & M & $46, X X$ & $S$ & $\begin{array}{l}\text { Hipospádia }+ \\
\text { criptorquidia bilateral }\end{array}$ & Homem XX & 8 \\
\hline 36 & $\mathrm{~F}$ & - & $S$ & Clitoromegalia & Deficiência 21 hidroxilase & 0,1 \\
\hline 37 & $\mathrm{~F}$ & - & $S$ & Pós-cirurgia corretiva & Deficiência 21 hidroxilase & 0,1 \\
\hline 38 & M & $46, X Y$ & S & Criptorquidia bilateral & Criptorquidia bilateral & 10 \\
\hline 39 & $\mathrm{~F}$ & CSP & $S$ & $\begin{array}{l}\text { Clitoromegalia }+ \\
\text { orifício urogenital único }\end{array}$ & Deficiência 21 hidroxilase & 0,1 \\
\hline 40 & M & $46, X Y$ & $S$ & $\begin{array}{l}\text { Micropênis + } \\
\text { criptorquidia bilateral }\end{array}$ & Micropênis + criptorquidia & 1 \\
\hline 41 & $\mathrm{~F}$ & $46, X X$ & S & $\begin{array}{l}\text { Clitoromegalia + } \\
\text { orifício urogenital único }\end{array}$ & Deficiência 21 hidroxilase & 0,1 \\
\hline 42 & M & $46, X Y$ del $18 q 22$ & $S$ & $\begin{array}{l}\text { Hipospádia }+ \\
\text { criptorquidia bilateral }\end{array}$ & Síndrome da deleção do $18 q$ & 5 \\
\hline 43 & M & $46, X Y$ & S & Hipospádia & Hipospádia & 12 \\
\hline 44 & $\mathrm{~F}$ & - & S & $\begin{array}{l}\text { Clitoromegalia + } \\
\text { orifício urogenital único }\end{array}$ & Deficiência 21 hidroxilase & 0,1 \\
\hline 45 & M & $46, X Y$ & S & Micropênis + hipospádia & Micropênis + hipospádia & 1 \\
\hline 46 & M & $46, X X$ & S & Micropênis + hipospádia & Homem XX & 35 \\
\hline 47 & $\mathrm{~F}$ & - & S & $\begin{array}{l}\text { Clitoromegalia + } \\
\text { fusão de grandes lábios }\end{array}$ & Deficiência 11 beta hidroxilase & 23 \\
\hline 48 & $\mathrm{~F}$ & - & S & $\begin{array}{l}\text { Clitoromegalia + } \\
\text { orifício urogenital único }\end{array}$ & Deficiência 21 hidroxilase & 0,4 \\
\hline 49 & M & $46, X Y$ inv 18 & S & $\begin{array}{l}\text { Hipospádia + criptorquidia } \\
\text { bilateral + bolsa escrotal } \\
\text { bífida }\end{array}$ & Deleção do 18q (suspeita) & 12 \\
\hline 50 & M & $46, X Y$ & $S$ & $\begin{array}{l}\text { Micropênis + } \\
\text { criptorquidia bilateral }\end{array}$ & Síndrome de Kallman & 29 \\
\hline 51 & $\mathrm{~F}$ & - & S & Pós-cirurgia corretiva & Deficiência 21 hidroxilase & 0,3 \\
\hline 52 & M & $46, X Y$ & S & Criptorquidia bilateral & Anorquia bilateral & 14 \\
\hline 53 & $\mathrm{~F}$ & - & S & $\begin{array}{l}\text { Clitoromegalia }+ \\
\text { orifício urogenital único }\end{array}$ & Deficiência 21 hidroxilase & 9 \\
\hline 54 & M & - & S & Criptorquidia bilateral & Síndrome de Prunne Belly & 0,1 \\
\hline 55 & M & $46, X Y$ & S & Micropênis & $\begin{array}{l}\text { Hipogonadismo } \\
\text { hipogonadotrófico isolado }\end{array}$ & 18 \\
\hline 56 & $\mathrm{~F}$ & $46, X X$ & S & $\begin{array}{l}\text { Clitoromegalia + } \\
\text { orifício urogenital único }\end{array}$ & Deficiência 21 hidroxilase & 3 \\
\hline 57 & $\mathrm{~F}$ & - & S & $\begin{array}{l}\text { Clitoromegalia + } \\
\text { orifício urogenital único }\end{array}$ & Deficiência 21 hidroxilase & 0,1 \\
\hline 58 & M & $46, X X$ & S & $\begin{array}{l}\text { Micropênis + } \\
\text { Criptorquidia bilateral }\end{array}$ & Hermafroditismo verdadeiro & 0,1 \\
\hline 59 & M & $46, X Y$ & S & Criptorquidia bilateral & Criptorquidia bilateral & 0,1 \\
\hline 60 & M & $46, X Y$ & S & Criptorquidia bilateral & Síndrome de Noonan (suspeita) & 9 \\
\hline 61 & M & $46, X Y$ & S & Criptorquidia bilateral & Disgenesia testicular & 0,1 \\
\hline 62 & M & - & S & Criptorquidia bilateral & Síndrome de Kallman & $?$ \\
\hline
\end{tabular}

GA: genitália ambígua; N: não; S: sim; CSP: cromatina sexual positiva; CSN cromatina sexual negativa. 
indivíduos submetidos à cirurgia de reconstrução genital, no entanto, foi entre um e dois a cada mil nascidos vivos $(0,1$ a $0,2 \%)$.

Em 2002, Sax ${ }^{(10)}$ criticou o estudo supracitado por ter incluído pacientes que habitualmente não são consideradas 'intersexos', por exemplo, os indivíduos com síndrome de Klinefelter, Turner e hiperplasia adrenal congênita de forma não-clássica. Sax concluiu que se fossem utilizados critérios mais rígidos para caracterizar os estados intersexuais, incluindo condições nas quais o sexo cromossômico fosse incompatível com o sexo fenotípico e os casos nos quais o sexo fenotípico não pudesse ser caracterizado como masculino ou feminino, a real prevalência desta afecção estaria em torno de $0,0018 \%$, quase 100 vezes menor do que o estimado por Blackless et al ${ }^{(7)}$.

Tais estudos mostram que a definição de genitália ambígua ainda é controversa e que a falta de consenso influencia na estimativa de sua freqüência. Em 2006, membros da Lawson Wilkins Pediatric Endocrine Society e da European Society for Pediatric Endocrinology publicaram um consenso em uma tentativa de modificar a nomenclatura e uniformizar o manejo destas afecções, propondo a utilização do termo Desordens da Diferenciação Sexual, em vez de intersexo, ambigüidade genital, hermafroditismo e pseudo-hermafroditismo, como se fazia até aquela época ${ }^{(6)}$. Essa nova nomenclatura incluiria não só a genitália ambígua, mas todos os casos de alterações na diferenciação sexual, mesmo aqueles cujo diagnóstico só seria percebido mais tarde, a partir de modificações na puberdade, presença de hérnia inguinal em meninas e infertilidade, entre outros.

Em 2007, especialistas brasileiros publicaram um comentário sobre este novo 'Consenso de Chicago', debatendo o impacto dessa nomenclatura na prática clínica. Apesar do objetivo nobre do consenso de tentar alterar a atual e estigmatizante classificação, os termos empregados não resolveriam totalmente a questão da terminologia. Conclui-se, portanto, que esta última nomenclatura deveria ser revista nas próximas edições do Consenso ${ }^{(11)}$.

No presente estudo, não foram incluídos vários casos de hiperplasia adrenal congênita por não apresentarem genitália ambígua ao nascimento (por exemplo, os casos de hiperplasia por defeito de 21 hidroxilase em pacientes do sexo masculino e os casos da forma não clássica), disgenesia gonadal pura $\mathrm{XY}$ cujo fenótipo era completamente feminino, entre outros diagnósticos. Se fossem utilizados esses novos conceitos, tais pacientes provavelmente seriam incluídos, assim como os casos de síndrome de Turner e de Klinefelter, freqüentemente acompanhados em nossos ambulatórios, o que aumentaria a freqüência do transtorno.

Por se tratar de um hospital de referência do Estado do Rio de Janeiro, o Iede possui um número significativo de casos. Ao calcular a prevalência dessa condição em nossa instituição, cujo número total de pacientes em acompanhamento está ao redor de 70 mil, observa-se prevalência de 0,88:10.000, um número bastante significativo, apesar do viés de amostragem, comparado à prevalência encontrada por $\operatorname{Sax}^{(10)}(0,0018 \%)$ e Castilla ${ }^{(8)}$ (1:20.000 nascimentos). De maneira similar à literatura mundial, o diagnóstico mais freqüente em nosso meio foi hiperplasia adrenal congênita, compreendendo quase $35 \%$ dos casos, seguida pelos quadros sindrômicos $(14,5 \%)$ e disgenesias gonadais $(9,7 \%)$.

Ao longo da história, o tema 'intersexo' foi motivo de preconceitos, dúvidas, debates e estudos ${ }^{(12)}$. Ainda hoje, os distúrbios da diferenciação sexual são um capítulo especial da prática clínica tanto por sua complexidade quanto pela carência de consenso em relação aos critérios diagnósticos e de manejo. Quanto mais soubermos sobre essas condições, tanto no que diz respeito à sua fisiopatologia, quanto à avaliação clínica e à história natural, melhor lidaremos com o paciente e, conseqüentemente, com sua saúde global, social e psicológica.

\section{Agradecimentos}

À doutora Vera Leal, chefe da Endocrinologia do Iede, ao doutor Ricardo Meirelles, diretor do Iede e ao doutor Luiz César Povoa, professor titular do curso de especialização em Endocrinologia e Metabologia da Pontifícia Universidade Católica do Rio de Janeiro (PUC-RJ), por tornarem este trabalho possível. 


\section{Referências bibliográficas}

1. Spinola-Castro AM. Importance of ethical and psychological features in the intersex management. Arq Bras Endocrinol Metabol 2005;49:46-59.

2. Danish RK. Intersex problems in the neonate. Indian J Ped 1982;49:555-75.

3. Guerra Junior G, Maciel-Guerra AT. Menino ou menina? Os distúrbios da diferenciação do sexo. $1^{\text {a }}$ ed. São Paulo: Manole; 2002.

4. White PC, Speiser PW. Congenital adrenal hyperplasia due to 21-hydroxylase deficiency. Endocr Rev 2000;21:245-91.

5. Lipay MV, Bianco B, Verreschi IT. Gonadal dysgenesis and tumors: genetic and clinical features. Arq Bras Endocrinol Metabol 2005;49:60-70.

6. Hughes IA, Houk C, Ahmed SF, Lee PA; LWPES Consensus Group; ESPE Consensus Group. Consensus statement on management of intersex disorders. Arch Dis Child 2006;91:554-63.
7. Blackless M, Charuvastra A, Derryck A, Fausto-Sterling A, Lauzanne K, Lee E. How sexually dimorphic are we? Review and synthesis. Am J Hum Biol 2000;12:151-66.

8. Castilla EE, Orioli IM, Lugarinho R, Dutra G. Epidemiology of ambiguous genitalia in South America. Am J Med Genet 1987;27:337-43.

9. Al-Mutair A, Iqbal M A, Sakati N, Ashwal A. Cytogenetics and etiology of ambiguous genitalia in 120 pediatric patients. Ann Saudi Med 2004;24:368-72.

10. Sax L. How common is intersex? A response to Anne Fausto-Sterling. J Sex Res 2002;39:174-8.

11. Damiani D, Guerra-Junior G. New definitions and classifications of the intersexual states: in which the Chicago Consensus has contributed to the state of the art? Arq Bras Endocrinol Metabol 2007;51:1013-7.

12. Mittwoch U. Sex determination in mythology and history. Arq Bras Endocrinol Metabol 2005;49:7-13. 